\title{
Numerical analysis of the resistance behavior of an electrostatically-induced graphene double junction
}

\author{
Paolo Marconcini · Massimo Macucci
}

Received: date / Accepted: date

\begin{abstract}
We present a numerical approach that we have developed in order to reproduce and explain the resistance behavior recently observed, as a function of the backgate voltage and of the position of a biased scanning probe, in a graphene flake in which a double $p$ - $n$ junction has been electrostatically induced. A simplified electrostatic model has been adopted to simulate the effect of gate voltages on the potential landscape, assuming for it a slow variation in space and using a simple capacitive model for the coupling between the electrodes and the graphene sheet. The transport analysis has then been performed with a solution of the Dirac equation in the reciprocal space coupled with a recursive scattering matrix approach. The efficiency of the adopted numerical procedure has allowed us to explore a wide range of possible potential landscapes and bias points, with the result of achieving a good agreement with available experimental data.
\end{abstract}

Keywords Numerical simulation · Graphene · Junctions - Dirac equation - Scanning probe measurement . Fabry-Pérot resonances

\section{Introduction}

Graphene [1-7] is one of the allotropes of carbon, along with fullerenes [8], carbon nanotubes [9, 10], graphite and diamond. It is made up of a two-dimensional hexagonal lattice of carbon atoms and is receiving a lot of

Paolo Marconcini and Massimo Macucci

Dipartimento di Ingegneria dell'Informazione

Università di Pisa

via Girolamo Caruso 16

I-56122 PISA, Italy

Tel.: +39050 2217537

Fax: +39050 2217522

E-mail: p.marconcini@iet.unipi.it attention as a result of its peculiar physical properties, and of its electrical, optical, mechanical and thermal characteristics, which make it interesting both for research in condensed matter physics and for many industrial applications.

In particular, a large effort is focusing on a possible use of graphene in the fabrication of nanoelectronic devices $[11,12]$, due to its high mobility and to its singleatom thickness, which makes lithographic patterning straightforward and control of its electrical properties possible by tuning the bias of suitable gates. Graphenebased electronic devices have been proposed for digital applications [13], for which several methods (for example, lateral confinement, doping [14], functionalization, the use of bilayer graphene biased via an orthogonal electric field) have been suggested to overcome the problem represented by the absence of an energy gap in bulk monolayer graphene. More promising applications of graphene have been suggested in the radiofrequency range [15], where the possibility of an electrostatic control of conductivity and the propagation of surface plasmon modes can be usefully exploited. Another successful electronic application of graphene is in the fabrication of sensors $[16,17]$, and, in particular, of chemical sensors, which can take advantage of its high surface to volume ratio.

Recently a "charge writing" technique, which can in general be adopted for the fabrication of prototype nanodevices, was applied in Cambridge to pattern the potential landscape in a graphene nanostructure [18]. A scanning probe was used to locally deposit charge into a dielectric layer situated over the graphene layer. In particular, in Ref. [19] a line of positive charge was injected into the PMMA (polymethyl methacrylate) layer deposited over a monolayer graphene flake. As a result, the graphene energy bands bend downwards under the 
charged region and a $p-n-p$ configuration is induced. A uniform bias can be applied through a backgate, separated from graphene by a dielectric layer. The sample was characterized by means of resistance and scanning gate microscopy measurements.

Here we describe the numerical method that we have developed to investigate such experimental results and present its application to a few representative simulations. We define an electrostatic model and a quantummechanical transport approach, which directly relate the potential energy at the graphene level to the resulting transport properties. This has allowed us to gain significant insight into the characteristics of the potential landscape resulting from the electrostatic action of the charge in the dielectric layer and from the presence of disorder.

\section{Analytical formulation}

In order to explore the dependence of the device conductance on the different potential details, we had to perform numerical simulations for a wide range of energy profiles and of bias voltages. Therefore, we decided to adopt an approximate but efficient method for the determination of the electrostatic landscape. Moreover, since we were interested in reproducing experiments, such as that of Ref. [19], in which graphene flakes a few microns wide are investigated, we decided to adopt an envelope-function $(\mathbf{k} \cdot \mathbf{p})$ description, instead of performing a full atomistic simulation. Therefore we modeled the transport properties of the sample using the Dirac-Weyl equation (well-known in relativistic quantum mechanics, where it describes the behavior of massless spin-1/2 particles), which represents the relation that the envelope functions have to satisfy in monolayer graphene, in the proximity of the Dirac points.

\subsection{Electrostatic simulation}

In principle, an exact simulation of the device would require a self-consistent solution of the electrostatic and transport equations. Indeed, the transport simulation requires the knowledge of the potential profile, which is in turn obtained solving the electrostatic equation. However, in order to perform the electrostatic analysis, it is necessary to know the spatial distribution of the mobile charges in the device, which can be derived from the solution of the transport equation. Therefore the electrostatic (Poisson) and transport (Dirac) equations are inherently coupled and should be iteratively solved using the solution of each of them as an input for the other one until a condition which reasonably satisfies both of them is reached [20,21]. In order to speed up our analysis, we have adopted a simplified approach, bypassing this self-consistent calculation under the hypothesis of a slow-varying potential profile and replacing the solution of the Poisson equation with a simple capacitive model for the device [22].

We have assumed to know the potential profile $U_{0}(\mathbf{r})$ in every point $\mathbf{r}$ of the flake for a given set of gate bias voltages $V_{G i 0}$ and we have computed its variation when different voltages $V_{G i}$ are applied to the existing gates (the backgate and the scanning probe, in our case).

In detail, if the voltages applied to the gates vary by $\Delta V_{G i}$ with respect to the reference values $V_{G i 0}$, they induce a change $\Delta \rho$ in the graphene charge density $\rho$ (with respect to the charge density $\rho_{0}$ which is present when the gates are biased at $\left.V_{G i 0}\right)$. Due to the finite density of states of graphene, this corresponds to a shift $\Delta U$ of the graphene potential $U$ with respect to $U_{0}$. The relation between the potential and the charge density is given (in quasi-equilibrium conditions) by

$$
\begin{aligned}
\rho(\mathbf{r}) & =e \int_{-\infty}^{U(\mathbf{r})} \operatorname{LDOS}(E, \mathbf{r})\left[1-f\left(E-E_{F}\right)\right] d E \\
& -e \int_{U(\mathbf{r})}^{+\infty} \operatorname{LDOS}(E, \mathbf{r}) f\left(E-E_{F}\right) d E
\end{aligned}
$$

where $e$ is the elementary charge, $\operatorname{LDOS}(E, \mathbf{r})$ is the local density of states at the energy $E$ and at the point $\mathbf{r}$, and $f\left(E-E_{F}\right)$ is the Fermi-Dirac occupation function ( $E_{F}$ is the Fermi level, set by the contacts). The first term in the r.h.s. is the contribution of the holes in the valence band [with occupation function $1-f\left(E-E_{F}\right)$ ], while the second term is the contribution of the electrons in the conduction band. The exact local density of states depends on the value of the wave function and thus its evaluation would require the solution of the Dirac equation; however, under the hypothesis of a slow varying potential, we can approximate it with the density of states shifted by the local value of the potential $U: \operatorname{LDOS}(E, \mathbf{r})=\operatorname{DOS}(E-U(\mathbf{r}))$, in such a way as to avoid the self-consistent solution of the electrostatic and transport equations. Since experiments are usually performed at low temperature, the Fermi-Dirac function can be approximated with a step function. Therefore, Eq. (1) becomes:

$$
\begin{aligned}
\rho_{0}+\Delta \rho & =e \int_{E_{F}}^{U_{0}+\Delta U} \operatorname{DOS}\left(E-U_{0}-\Delta U\right) d E \\
& =\operatorname{sign}\left(U_{0}+\Delta U-E_{F}\right) \frac{e\left(U_{0}+\Delta U-E_{F}\right)^{2}}{\pi\left(\hbar v_{F}\right)^{2}}
\end{aligned}
$$

where $\rho$ and $U$ have been substituted by $\rho_{0}+\Delta \rho$ and $U_{0}+\Delta U$, and we have exploited the expression of the 
density of states in unconfined (or very large ribbons of) monolayer graphene: $\operatorname{DOS}(E)=2|E| /\left(\pi\left(\hbar v_{F}\right)^{2}\right)$.

In order to reduce the complexity of the problem, the relation between the charge in the device and the gate voltages can be approximately modeled through the electrostatic capacitances (per unit area) $C_{G i}(\mathbf{r})$ which couple the gates to each point $\mathbf{r}$ of the graphene layer. Therefore the following relation can be written, linking the charge with the voltage variations in the device:

$\Delta \rho=\sum_{i} C_{G i}\left(\frac{\Delta U}{-e}-\Delta V_{G i}\right)$,

where $\Delta \rho$ is expressed as the sum of the variations of the charge on the graphene side of all the capacitors.

By substituting Eq. (3) into Eq. (2) we obtain a second-order equation that can be analytically solved, obtaining the value of $\Delta U$. This calculation has been repeated for all the points $\mathbf{r}$ of the flake, in such a way as to obtain the profiles $U(\mathbf{r})$ and $\rho(\mathbf{r})$ in the device.

\subsection{Transport simulation and validation of the method}

Then the potential profile $U(\mathbf{r})$ has been used to compute the conductance of the sample. In order to reach this result, we have used our envelope-function based transport code for graphene armchair ribbons [23, 24], based on the solution of the Dirac equation (which represents the envelope-function transport equation for monolayer graphene [25]) in the device (the exact detail of the edges has a very small effect on the behavior of ribbons as wide as those we have considered).

In a graphene armchair ribbon, the electron wave function can be written as a linear combination of the $2 p^{z}$ orbitals $\varphi$ of all the carbon atoms centered at the lattice points $\mathbf{R}_{j}$ of the two inequivalent sublattices $A$ and $B$

$\psi(\mathbf{r})=\sum_{j=A, B} \sum_{\mathbf{R}_{j}} \psi_{j}\left(\mathbf{R}_{j}\right) \varphi\left(\mathbf{r}-\mathbf{R}_{j}\right)$.

Since atoms of both sublattices appear at the edges of the ribbon, both $\psi_{A}$ and $\psi_{B}$ have to vanish there (boundary conditions). The functions $\psi_{A}(\mathbf{r})$ and $\psi_{B}(\mathbf{r})$ are related to the 4 graphene envelope functions $F$, which satisfy the Dirac transport equation, by

$$
\begin{aligned}
& \psi_{A}(\mathbf{r})=e^{i \mathbf{K} \cdot \mathbf{r}} F_{A}^{\mathbf{K}}(\mathbf{r})-i e^{i \mathbf{K}^{\prime} \cdot \mathbf{r}} F_{A}^{\mathbf{K}^{\prime}}(\mathbf{r}) \\
& \psi_{B}(\mathbf{r})=i e^{i \mathbf{K} \cdot \mathbf{r}} F_{B}^{\mathbf{K}}(\mathbf{r})+e^{i \mathbf{K}^{\prime} \cdot \mathbf{r}} F_{B}^{\mathbf{K}^{\prime}}(\mathbf{r})
\end{aligned}
$$

( $\mathbf{K}$ and $\mathbf{K}^{\prime}$ are the two inequivalent Dirac points of graphene, where the conduction and valence bands are degenerate).
In our simulations we subdivide the device into a series of thin cascaded sections. Their length is chosen in such a way that within each of them the variation of the potential in the transport direction $x$ is negligible and thus the potential approximately depends only on $y$. In every section, each envelope function can be written in the form $F(x, y)=e^{i \kappa_{x} x} \Phi(y)$, i.e. as the product of a confined component $\Phi$ in the transverse direction $y$ and of a plane wave propagating, with wave vector $\kappa_{x}$, along the transport coordinate $x$. With these positions, the Dirac equation with the exact boundary conditions becomes:

$$
\left\{\begin{array}{l}
\left(\boldsymbol{\sigma}_{x} f(y)+\boldsymbol{\sigma}_{z} \partial_{y}\right) \boldsymbol{\varphi}^{\mathbf{K}}(y)=-\kappa_{x} \boldsymbol{\varphi}^{\mathbf{K}}(y) \\
\left(\boldsymbol{\sigma}_{x} f(y)-\boldsymbol{\sigma}_{z} \partial_{y}\right) \boldsymbol{\varphi}^{\mathbf{K}^{\prime}}(y)=-\kappa_{x} \boldsymbol{\varphi}^{\mathbf{K}^{\prime}}(y) \\
\boldsymbol{\varphi}^{\mathbf{K}}(0)=\boldsymbol{\varphi}^{\mathbf{K}^{\prime}}(0) \\
\boldsymbol{\varphi}^{\mathbf{K}}(\tilde{W})=e^{i 2 \tilde{K} \tilde{W}} \boldsymbol{\varphi}^{\mathbf{K}^{\prime}}(\tilde{W}) .
\end{array}\right.
$$

In this system $\partial_{y}=d / d y, \boldsymbol{\sigma}_{x}, \boldsymbol{\sigma}_{y}$ and $\boldsymbol{\sigma}_{z}$ are the Pauli matrices, $\varphi^{\mathbf{K}}=\left[\Phi_{A}^{\mathbf{K}}, \Phi_{B}^{\mathbf{K}}\right]^{T}, \varphi^{\mathbf{K}^{\prime}}=i\left[\Phi_{A}^{\mathbf{K}^{\prime}}, \Phi_{B}^{\mathbf{K}^{\prime}}\right]^{T}$, $\tilde{W}$ is the effective width of the ribbon, $f(y)=(U(y)-$ $E) /\left(\hbar v_{F}\right)$ (with $E$ the total energy of the mobile charges, $\hbar$ the reduced Planck constant and $v_{F}$ the Fermi velocity of graphene), and $\tilde{K}=K-\operatorname{round}(K \tilde{W} / \pi) \pi / \tilde{W}$ (where $K=|\mathbf{K}|=4 \pi /(3 a)$, $a$ being the lattice constant of graphene).

While the application of a standard finite difference method to this differential system suffers from the socalled fermion doubling problem, with the appearance of spurious results, a very efficient solution can be obtained if we define, within the domain $[0,2 \tilde{W}]$, the function $[23,26]$

$\boldsymbol{\varphi}(y)= \begin{cases}\boldsymbol{\varphi}^{\mathbf{K}}(y) & \text { for } y \in[0, \tilde{W}] \\ e^{i 2 \tilde{K} \tilde{W}} \boldsymbol{\varphi}^{\mathbf{K}^{\prime}}(2 \tilde{W}-y) & \text { for } y \in[\tilde{W}, 2 \tilde{W}] .\end{cases}$

With this definition, the problem (6) becomes:

$\left\{\begin{array}{l}{\left[\boldsymbol{\sigma}_{z} \partial_{y}+\boldsymbol{\sigma}_{x} f(\tilde{W}-|\tilde{W}-y|)\right] \boldsymbol{\varphi}(y)=-\kappa_{x} \boldsymbol{\varphi}(y)} \\ e^{-i 2 \tilde{K} \tilde{W}} \boldsymbol{\varphi}(2 \tilde{W})=\boldsymbol{\varphi}(0)\end{array}\right.$

which represents a differential problem with periodic boundary conditions for the function $e^{-i \tilde{K} y} \varphi(y)$. This problem can be solved in the reciprocal space, substituting their Fourier expansions to $e^{-i \tilde{K} y} \boldsymbol{\varphi}(y)$ and $f(\tilde{W}-|\tilde{W}-y|)$, extended by periodicity with period $2 \tilde{W}$. Projecting onto the basis functions of the Fourier expansion, the system becomes a linear eigenproblem in the Fourier coefficients of $e^{-i \tilde{K} y} \boldsymbol{\varphi}(y)$, with eigenvalues $\kappa_{x}$. Once we have computed the Fourier coefficients of $f(\tilde{W}-|\tilde{W}-y|)$, this eigenproblem can be efficiently solved (after limiting its size with a proper frequency cut-off). 
In this way we compute the transport modes in all the sections into which we have divided the device. Then we have to enforce the continuity of the wave function along the interface between adjacent sections. This can be done for each of the modes impinging, from the left and from the right, against the interface (modematching technique). By projecting the resulting set of continuity equations onto a proper basis of sine functions, we obtain a linear system in the transmission and reflection coefficients, which relate the modes in the two adjacent sections and which represent the elements of the corresponding scattering matrix. Once we have found the scattering matrices of all the regions making up the device, we can iteratively compose them, obtaining the scattering matrix from the entrance to the exit of the graphene ribbon and thus, as a submatrix, the overall transmission matrix t. Finally, the conductance $G$ of the sample can be computed from $\mathbf{t}$ using the Landauer-Büttiker formula. All of the transport calculations can be performed directly in terms of the Fourier coefficients computed within each section, thereby increasing the efficiency of the numerical algorithm.

In Fig. 1 we compare the results of our approximate method with those obtained with the open-source simulation code NanoTCAD ViDES [27,28], which selfconsistently solves the Poisson equation and the transport equation, using an atomistic, tight-binding, model for the structure and a non-equilibrium Green's function approach for the transport analysis. In order to make the full self-consistent atomistic simulation possible within a reasonable time, we have simulated a structure containing a limited number of atoms: a $4 \mathrm{~nm}$ wide and $10 \mathrm{~nm}$ long armchair graphene ribbon. We have also included the effect of an underlying backgate, separated from the ribbon by a $1 \mathrm{~nm}$ thick $\mathrm{SiO}_{2}$ layer and biased at a voltage $V_{G}$. The ribbon is covered with a $1 \mathrm{~nm}$ layer of $\mathrm{SiO}_{2}$, in which a uniform charge in the amount of $10^{26}$ elementary charges per $\mathrm{m}^{3}$ has been injected in a transverse stripe that is $1 \mathrm{~nm}$ wide and $6 \mathrm{~nm}$ long (see Fig. 1(a)-(b)). We have first used ViDES to compute the potential landscape $U_{0}(\mathbf{r})$ in the graphene ribbon when a reference voltage $V_{G}=0.5 \mathrm{~V}$ is applied to the backgate. Then, starting from this profile $U_{0}$, we have used our approximate method to compute the potential profile $U$ when the voltages $V_{G}=-0.5 \mathrm{~V}$ and $V_{G}=-3 \mathrm{~V}$ are applied to the backgate. We have compared these two profiles with those obtained directly using ViDES for the two same values of the gate voltage. The comparison between the results obtained along the transport direction using our approximate method and the self-consistent quantum simulator of ViDES is shown in Fig. 1(c). Even though in our method we exploit the analytical expression of the density of states
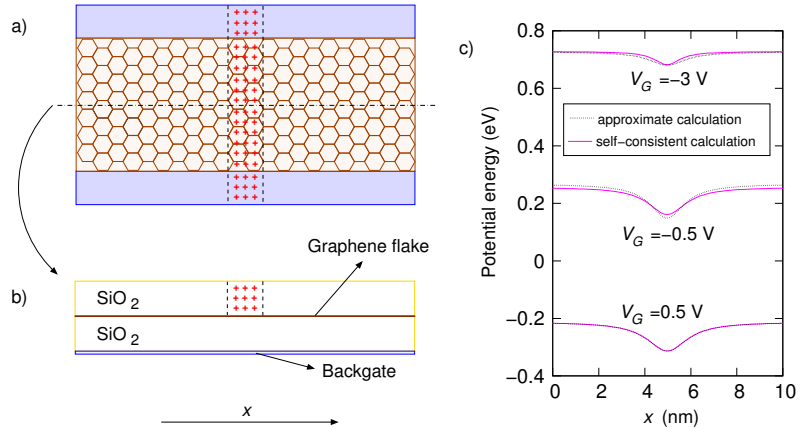

Fig. 1 Top (a) and cross-section (b) view of the small structure for which the potential profiles obtained with our approximate approach have been compared with those obtained with the atomistic self-consistent solver NanoTCAD ViDES (c)

for wide ribbons, the agreement already appears rather good, and certainly improves for larger graphene flakes.

\section{Numerical results}

Here we present some numerical results obtained from the simulation of a structure close to the one investigated in Ref. [19], in which a graphene flake with a width of approximately $2.5 \mu \mathrm{m}$ is deposited on a $300 \mathrm{~nm}$ thick silicon oxide dielectric layer on top of a highly doped silicon substrate, which acts as a backgate. A $100 \mathrm{~nm}$ thick layer of PMMA is assumed to be on top of the graphene flake, with a line of positive charge injected into it in the transverse direction (with respect to charge transport). As mentioned in the introduction, as a consequence of the electrostatic action of the deposited charge, a cavity-shaped potential profile for electrons appears.

We have modeled the flake with a $2.5 \mu \mathrm{m}$ wide armchair ribbon and in the longitudinal direction we have considered only the $1 \mu \mathrm{m}$ long part nearest to the line of charge. The Fermi energy (established by the contacts) has been assumed as the reference value $E_{F}=0$. For the capacitance (per unit area) coupling the backgate to the graphene flake we have assumed a value $C_{B G}=0.1151 \mathrm{mF} / \mathrm{m}^{2}$, which corresponds to a parallel plate capacitor with a $300 \mathrm{~nm}$ thick $\mathrm{SiO}_{2}$ dielectric. In the transport calculation we have divided the overall flake into $4 \mathrm{~nm}$ long sections and we have considered 330 modes (after verifying that a further increase did not determine a substantial variation of the results). The Fourier coefficients of $f(\tilde{W}-|\tilde{W}-y|)$ have been computed by means of a Fast Fourier Transform algorithm performed on 2048 spatial samples.

We have initially focused on the influence of the shape of the cavity on the dependence of the sample resistance on backgate voltage. In detail, an increase in 

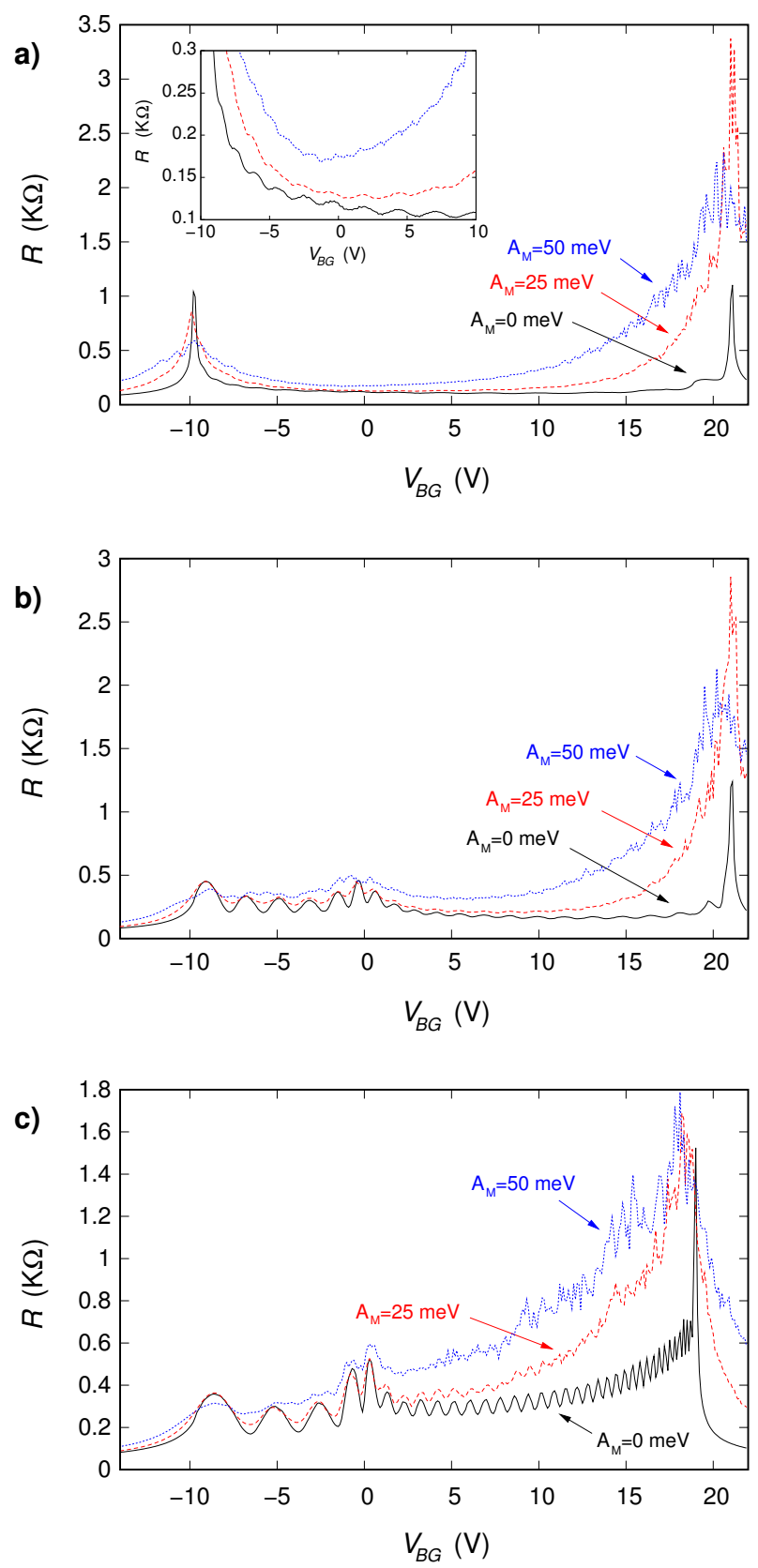

Fig. 2 Behavior of the resistance as a function of the backgate voltage $V_{B G}$, for a cavity with square (a), parabolic (b), and Lorentzian (c) shape at $V_{B G}=0$, in the presence of disorder that has been modeled by summing Gaussian functions with an amplitude uniformly distributed in the range $\left[-A_{M}, A_{M}\right]$. In the case of the square cavity, we report a detail of the main plot in the inset.

the value of $V_{B G}$ involves a decrease of the potential energy $U$ at the graphene level. For low (high) values of $V_{B G}$ the potential $U$ takes on values higher (lower) than the Fermi energy $E_{F}$ of the mobile charges everywhere. In such a situation, carriers do not experience any scattering against the potential profile, and the resistance of the sample is low. Instead, for intermediate values of $V_{B G}$ carriers scatter against the cavity-shaped potential profile and the sample can be seen as two $p$ regions (where $E_{F}<U$ ) separated by an $n$ region (where $\left.E_{F}>U\right)$, corresponding to the cavity. For these values of $V_{B G}$ the interference between the transmitted and the reflected wave (for the nonzero values of the incident angle, for which a reflection takes place) give rise to Fabry-Pérot resonances in the behavior of the resistance as a function of $V_{B G}$ [29]. We have investigated several cavity shapes, widths and depths. The results for three different cavity shapes are reported in Figs. 2(a-c). In Fig. 2(a) (black solid curve for a "clean" potential landscape, i.e. without random impurities) we show the dependence of the device resistance on backgate voltage for a $180 \mathrm{~nm}$ wide and $210 \mathrm{meV}$ deep square potential well: there are Fabry-Pérot oscillations, but they are rather weak and just barely visible (see the inset of Fig. 2(a)). The effect of a potential well with a parabolic profile is reported with the black solid curve in Fig. 2(b), and shows more apparent Fabry-Pérot fluctuations, in particular for negative backgate voltages. A much stronger effect can be seen in Fig. 2(c), for a Lorentzian shape of the potential defining the well. Also in this case the curve to be considered is the black solid one, referring to the case of a smooth potential. In Figs. 2(a-c) we report also the effect of more realistic potential landscapes, in which the contribution of randomly positioned impurities has been included. The perturbation of the potential due to randomly located dopants and charge impurities can be approximately evaluated by summing up terms representing the screened potential due to each impurity. In our simulations we have considered a defect density equal to $5 \times 10^{11} \mathrm{~cm}^{-2}$ and a Gaussian shape, with standard deviation $5 \mathrm{~nm}$ and a random amplitude, uniformly distributed around zero, for the electrostatic contribution of each donor on the graphene layer. The red dashed curves, obtained considering the amplitude of each term uniformly distributed in the range [-25 meV, $25 \mathrm{meV}$ ], already exhibit a significant suppression of the Fabry-Pérot oscillations, while increasing the amplitude range to $[-50 \mathrm{meV}, 50 \mathrm{meV}]$ the oscillations are substantially canceled for most values of the backgate voltage (blue dotted curves). The square, parabolic or Lorentzian shape of the cavity is assumed at zero backgate voltage; at any other backgate voltage the actual behavior of the potential is obtained from the previously described approach to the solution of the electrostatic problem.

In Figs. 3(a-c) we report the longitudinal variation of the potential for the three cases, at backgate voltages of $-10 \mathrm{~V}, 0 \mathrm{~V}$ and $10 \mathrm{~V}$. 

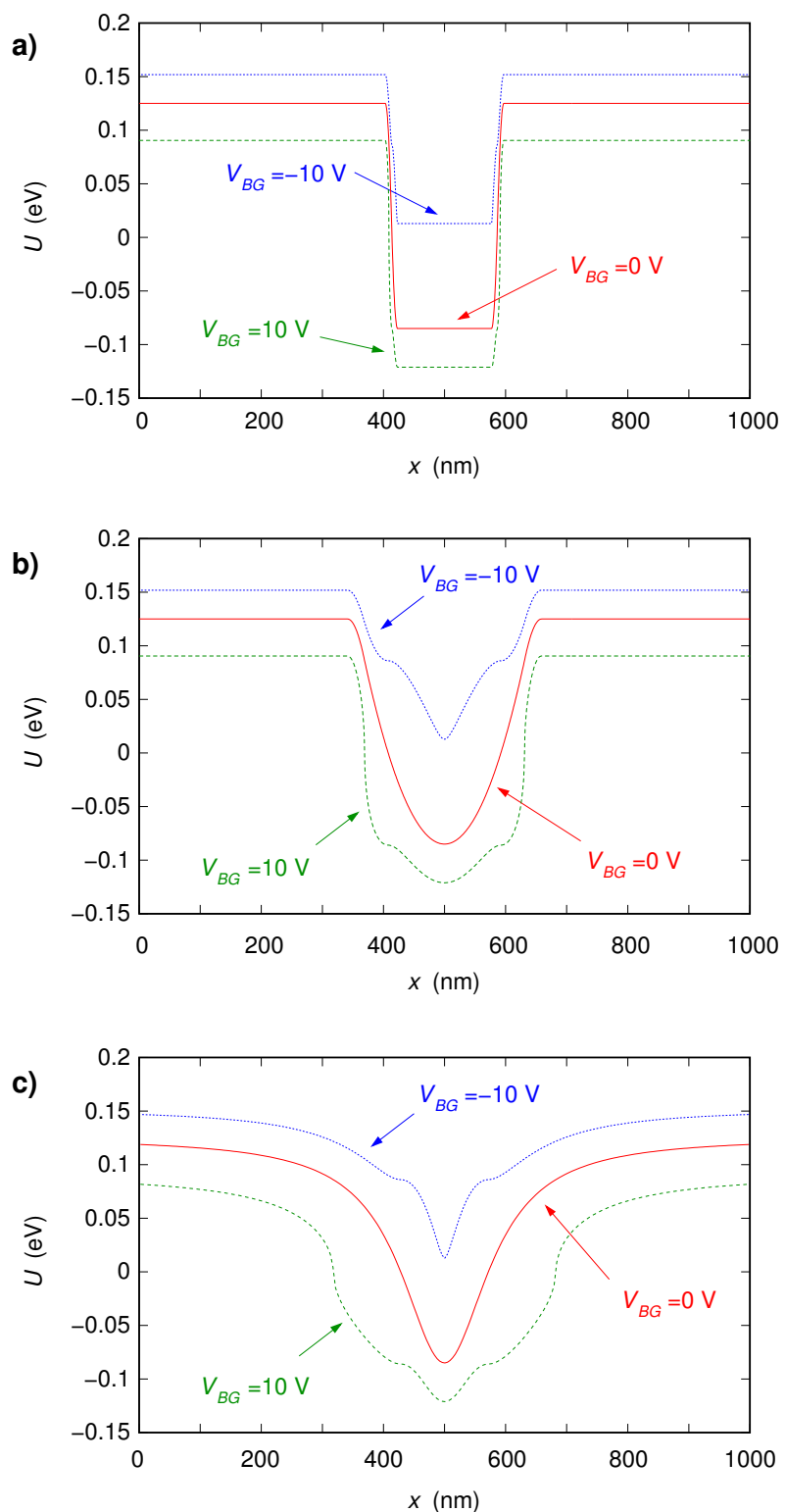

Fig. 3 Longitudinal section of the potential profile for three different cavity shapes [square (a), parabolic (b) and Lorentzian (c) at $V_{B G}=0$ ], reported for three different values of the voltage $V_{B G}$ applied to the backgate $(-10 \mathrm{~V}, 0 \mathrm{~V}$ and $10 \mathrm{~V})$.

Comparing the results obtained from our calculations with the data reported in Ref. [19], we have found that the longitudinal cavity shape at $V_{B G}=0$ that best fits the experimentally measured separation between resonance peaks is a Lorentzian $U_{0}=a-b /(1+((x-$ $\left.\left.x_{0}\right) / c\right)^{2}$ ), with $a=125 \mathrm{meV}, b=210 \mathrm{meV}, c=90 \mathrm{~nm}$, $x_{0}$ being the coordinate at the center of the cavity.

Our numerical approach can be applied also to the analysis of scanned probe spectroscopy experiments [30, 31]. In such experiments a negatively biased probe is scanned at a fixed distance over the sample. For each

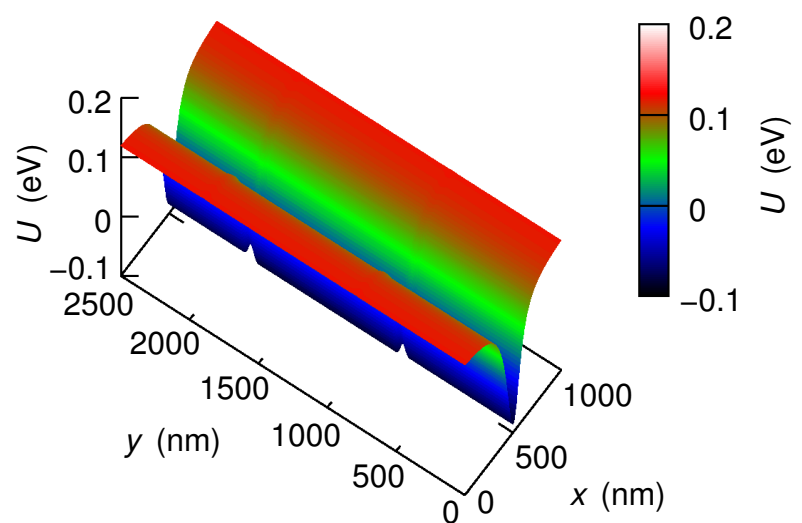

Fig. 4 Potential profile in the flake for $V_{B G}=0$, with 3 parallel cavities.

position of the probe the resistance of the graphene sample, modulated by the action of the probe itself, is recorded, leading to the creation of a two-dimensional map. A numerical simulation of such a map involves a rather large computational effort, because a complete transport and electrostatic problem has to be solved for each position of the probe (although some effort can be spared with a proper numerical treatment [32]). Thus we have adapted our simplified approach to the solution of the electrostatic problem to include also the effect of the biased probe. This has been achieved through a spatially variable capacitance: in detail, we have assumed, between the probe and the generic point $\mathbf{r}$ of the graphene flake, a capacitance (per unit area) with a Lorentzian dependence on the distance $d$ between the projection on the flake of the tip apex and the point $\mathbf{r}: C_{P}(\mathbf{r})=C_{P M} /\left(1+(d / D)^{2}\right)$. In the simulations for which we report the results, we have assumed $C_{P M}=0.12 \mathrm{mF} / \mathrm{m}^{2}$ and $D=110 \mathrm{~nm}$. These values have been obtained solving the Laplace equation between the graphene flake and a conic probe (with an opening angle of 30 degrees and a spherical tip, with radius $100 \mathrm{~nm}$ ) located at a distance of $110 \mathrm{~nm}$, with the correct material stack. We have considered a scan over a $40 \times 100$ uniform grid of points across the sample.

In particular, a good approximation of the scanning probe measurements reported in Ref. [19] has been obtained considering the subdivision of the previously chosen Lorentzian cavity into multiple parallel cavities. The separation walls, with a height equal to about $1 / 10$ of the cavity depth, can be the result of the disorder of charged impurities in the substrate or in the charge injected in the PMMA. We have modeled this potential multiplying the longitudinal Lorentzian profile by a proper factor depending only on the transverse coordinate $y$. We have connected the bottom of each cavity to the top of the separation wall through the series of two arcs of parabola with opposite curvature, chosen in such 


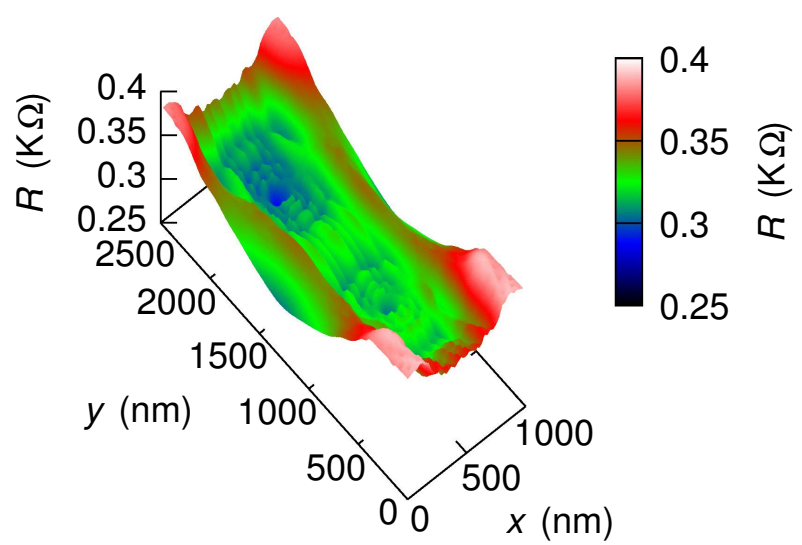

Fig. 5 Resistance of the sample reported as a function of the position of a biased probe kept at a fixed distance over the graphene sheet, for the potential profile (represented by 3 parallel cavities) shown in Fig. 4.

a way as to avoid discontinuities in the potential and in its first derivative. More in detail, the region connecting the points with transversal coordinate $y_{\text {bottom }}$ (at potential $\left.V_{\text {bottom }}\right)$ to the points with coordinate $y_{\text {top }}=$ $y_{\text {bottom }}+2 \Delta$ (at potential $\left.V_{\text {top }}\right)$ has been described with a parabola $V_{\text {bottom }}+c\left(y-y_{\text {bottom }}\right)^{2}$ between $y_{\text {bottom }}$ and $y_{\text {bottom }}+\Delta$, and a parabola $V_{\text {top }}-c\left(y-y_{\text {top }}\right)^{2}$ between $y_{\text {bottom }}+\Delta$ and $y_{\text {top }}$, with $\Delta=\sqrt{\left(V_{\text {top }}-V_{\text {bottom }}\right) /(2 c)}$.

In Fig. 4 we represent the overall potential profile, with 3 parallel cavities, for $V_{B G}=0$. In Fig. 5 we report the resistance, as a function of the position of the probe, for a probe bias of $-10 \mathrm{~V}$.

The biased probe, perturbing the potential profile in the graphene flake, modifies the scattering experienced by the mobile charges and thus the resistance of the sample. In these simulations we have considered 3 parallel cavities, with different widths: $795 \mathrm{~nm}, 1105 \mathrm{~nm}$, and $600 \mathrm{~nm}$, and bottom values: $-0.085 \mathrm{eV},-0.088 \mathrm{eV}$, and $-0.083 \mathrm{eV}$, respectively. We observe the appearance

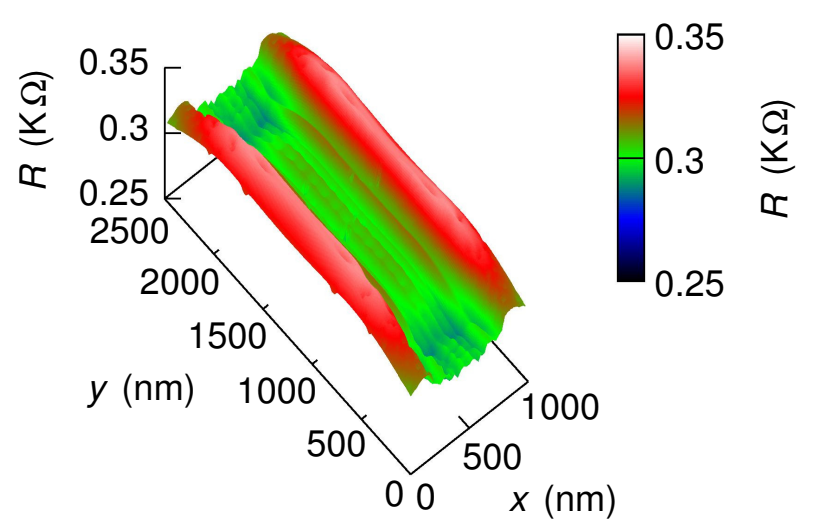

Fig. 6 Resistance of the sample reported as a function of the position of a biased probe kept at a fixed distance over the graphene sheet, for a potential profile with a single cavity.

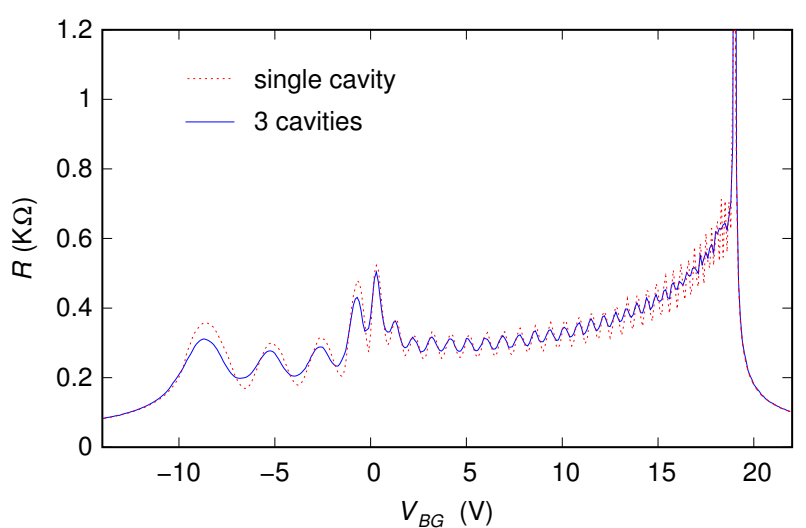

Fig. 7 Behavior of the resistance as a function of the backgate voltage, obtained considering a single cavity (red dotted curves) or 3 parallel cavities (blue solid curves).

of circular features analogous to those experimentally observed in Ref. [19].

In Fig. 6, instead, we report the computed resistance for an analogous two-dimensional scan, but for a potential profile with a single cavity, which exhibits much less structure. The comparison between Fig. 5 and Fig. 6 confirms that the division into parallel cavities leads to a significant variation of the effect of the probe on the sample resistance and to scanning probe maps that better fit the experimental results in Ref. [19].

Finally, in Fig. 7 we compare the behavior of the resistance as a function of the backgate voltage for the case of a single cavity and of 3 parallel cavities. In this case, the effect of the division into multiple cavities is very limited, just a mild reduction of the Fabry-Pérot resonances.

\section{Conclusion}

We have performed a numerical analysis of the transport properties of a graphene sample with a cavityshaped potential profile in the direction of current propagation. Using a simplified electrostatic model to maximize the numerical efficiency and an envelope-function based transport simulator, we have been able to reproduce the resistance behavior that has been experimentally measured for this device, as a function of the voltage applied to a backgate and of the position of a negatively charged probe scanned at a fixed distance from the flake. In particular, performing an extensive exploration of the parameter space and comparing the obtained results with available experimental data, we have been able to understand the details of the potential profile electrostatically induced onto the graphene sheet and the role they have on the behavior of the resistance. 


\section{References}

1. Castro Neto, A. H., Guinea, F., Peres, N. M. R., Novoselov, K. S., Geim, A. K.: The electronic properties of graphene. Rev. Mod. Phys. 81, 109-162 (2009)

2. Novoselov, K. S., Fal'ko, V. I., Colombo, L., Gellert, P. R., Schwab, M. G., Kim, K.: A roadmap for graphene. Nature 490, 192-200 (2012)

3. Avouris, P., Dimitrakopoulos, C.: Graphene: synthesis and applications. Mater. Today 15, 86-97 (2012)

4. Katsnelson, M. I.: Graphene: Carbon in Two Dimensions. Cambridge University Press, Cambridge (2012)

5. Foa Torres, L. E. F., Roche, S., Charlier, J.-C.: Introduction to Graphene-Based Nanomaterials: From Electronic Structure to Quantum Transport. Cambridge University Press, Cambridge (2014)

6. Wolf, E. L.: Graphene: A New Paradigm in Condensed Matter and Device Physics. Oxford University Press, Oxford (2013)

7. Connolly, M. R., Puddy, R. K., Logoteta, D., Marconcini, P., Roy, M., Griffiths, J. P., Jones, G. A. C., Maksym, P. A., Macucci, M., Smith, C. G.: Unraveling Quantum Hall Breakdown in Bilayer Graphene with Scanning Gate Microscopy. Nano Lett. 12, 5448-5454 (2012)

8. Kroto, H. W., Walton, D. R. M.: The Fullerenes: New Horizons for the Chemistry, Physics and Astrophysics of Carbon. Cambridge University Press, Cambridge (1993)

9. Saito, R., Dresselhaus, G., Dresselhaus, M. S.: Physical Properties of Carbon Nanotubes. Imperial College Press, London (1998)

10. Ganin, A. A., Bityutskaya, L. A., Bormontov, E. N.: Atomic configuration, band structure and stability of fluorinated carbon nanotubes. International Journal of Materials 1, 93-98 (2014)

11. Raza, H.: Graphene Nanoelectronics: Metrology, Synthesis, Properties and Applications. Springer, Berlin (2012)

12. Schwierz, F.: Graphene transistors. Nature Nanotech. 5, 487-496 (2010)

13. Iannaccone, G., Fiori, G., Macucci, M., Michetti, P., Cheli, M., Betti, A., Marconcini, P.: Perspectives of graphene nanoelectronics: probing technological options with modeling. IEEE International Electron Devices Meeting (IEDM) 2009, IEEE Conference Proceedings, 10.4.110.4.4 (2009). doi: 10.1109/IEDM.2009.5424376

14. Marconcini, P., Cresti, A., Triozon, F., Fiori, G., Biel, B., Niquet, Y.-M., Macucci, M., Roche, S.: Atomistic BoronDoped Graphene Field-Effect Transistors: A Route toward Unipolar Characteristics. ACS Nano 6, 7942-7947 (2012)

15. Liao, L., Duan, X.: Graphene for radio frequency electronics. Mater. Today 15, 328-338 (2012)

16. Hill, E. W.: Graphene Sensors. IEEE Sensors J. 11, 3161$3170(2011)$

17. Marconcini, P., Macucci, M.: Symmetry-dependent transport behavior of graphene double dots. J. Appl. Phys. 114, 163708-1-163708-6 (2013)
18. Connolly, M. R., Herbschleb, E. D., Puddy, R. K., Roy, M., Anderson, D., Jones, G. A. C., Maksym, P., Smith, C. G.: Reading and writing charge on graphene devices. Appl. Phys. Lett. 101, 023505-1-023505-4 (2012)

19. Herbschleb, E. D., Puddy, R. K., Marconcini, P., Griffiths, J. P., Jones, G. A. C., Macucci, M., Smith, C. G., Connolly, M. R.: Direct Imaging of Coherent Quantum Transport in Graphene Heterojunctions. arXiv:1408.1925

20. Marconcini, P.: The role of the choice of the physical model in the optimization of nanoelectronic device simulators. International Journal of Circuits, Systems and Signal Processing 7, 173-180 (2013)

21. Marconcini, P.: A fast approach to the simulation of silicon nanowire transistors. International Journal of Circuits, Systems and Signal Processing 7, 206-213 (2013)

22. Marconcini, P., Macucci, M.: Approximate calculation of the potential profile in a graphene-based device. IET Circuits, Devices \& Systems (2014). doi: 10.1049/ietcds.2014.0003.

23. Logoteta, D., Marconcini, P., Bonati, C., Fagotti, M., Macucci, M.: High-performance solution of the transport problem in a graphene armchair structure with a generic potential. Phys. Rev. E 89, 063309-1-063309-11 (2014)

24. Marconcini, P., Logoteta, D., Macucci, M.: Sinc-based method for an efficient solution in the direct space of quantum wave equations with periodic boundary conditions. J. Appl. Phys. 114, 173707-1-173707-10 (2013)

25. Marconcini, P., Macucci, M.: The $\mathbf{k} \cdot \mathbf{p}$ method and its application to graphene, carbon nanotubes and graphene nanoribbons: the Dirac equation. La Rivista del Nuovo Cimento 34, No. 8-9, 489-584 (2011)

26. Fagotti, M., Bonati, C., Logoteta, D., Marconcini, P., Macucci, M.: Armchair graphene nanoribbons: $\mathcal{P} \mathcal{T}$ symmetry breaking and exceptional points without dissipation. Phys. Rev. B 83, 241406-1-241406-4 (2011)

27. Code is available at http://nanohub.org/resources/vides/. doi: 10254/nanohub-r5116.5

28. Fiori, G., Iannaccone, G.: Multiscale Modeling for Graphene-Based Nanoscale Transistors. Proceedings of the IEEE 101, 1653-1669 (2013)

29. Shytov, A. V., Rudner, M. S., Levitov, L. S.: Klein Backscattering and Fabry-Pérot Interference in Graphene Heterojunctions. Phys. Rev. Lett. 101, 156804-1-156804-4 (2008)

30. Topinka, M. A., LeRoy, B. J., Westervelt, R. M., Shaw, S. E. J., Fleischmann, R., Heller, E. J., Maranowski, K. D., Gossard, A. C.: Coherent branched flow in a twodimensional electron gas. Nature 410, 183-186 (2001)

31. Crook, R., Smith, C. G., Simmons, M. Y., Ritchie, D. A.: One-dimensional probability density observed using scanned gate microscopy. J. Phys.: Condens. Matter 12, L735-L740 (2000)

32. Metalidis, G., Bruno, P.: Green's function technique for studying electron flow in two-dimensional mesoscopic samples. Phys. Rev. B 72, 235304-1-235304-9 (2005) 